\title{
The prevalence of hrHPV among the isolated community in the Highland of Bario, Sarawak, East Malaysia
}

\section{Abigail Rembui Jerip}

Universiti Pertanian Malaysia Institut Teknologi Maju

\section{Mardiana Kipli}

Universiti Malaysia Sarawak

Cheng-Siang Tan ( $\nabla$ cstan@unimas.my )

Universiti Malaysia Sarawak https://orcid.org/0000-0001-6312-4551

\section{Research article}

Keywords: Cervical cancer, human papillomavirus, prevalence, hrHPV

Posted Date: November 8th, 2019

DOl: https://doi.org/10.21203/rs.2.17048/v1

License: (c) (i) This work is licensed under a Creative Commons Attribution 4.0 International License.

Read Full License 


\section{Abstract}

Background Malaysia has introduced the free Pap smear as the primary cervical cancer screening program since the 1960 s but has only achieved the national coverage of $22 \%$. The coverage would be much lower in the rural communities especially in Sarawak where many villages are still inaccessible by road, and communities are living in low resource settings. In this study, we have evaluated Qiagen's careHPV system as a point-of-care HPV diagnostic test together with the visualization using acetic acid (VIA) in the isolated community in the Highland of Bario which are predominantly from the Kelabit ethnicity. Methods Women attending the cervical cancer outreach program were recruited. Consent was obtained and a cervical swab was clinician-collected and screened for High-risk HPV DNA using the careHPV system. Cervical examination using VIA was offered to all women age $<50$-year. hrHPV positive women were recruited during their follow-up session and the second cervical swab was collected for HPV genotyping using consensus primer-nested PCR to elucidate the infecting HPV genotypes. Results Seventy-five women from the age of 27-83 (mean age 55) attended the cervical cancer screening clinic and $8 \%(n=6 / 75)$ of them were positive for hrHPV. Only 33 women were screened using VIA and $21.2 \%$ $(n=7 / 33)$ were found to be positive. A majority of the women (58.6\%) were above the age of 50-year and not recommended for VIA to be performed. Thus, it was not feasible to correlate the results obtained from the HPV DNA test and VIA. Only four hrHPV positive women returned for follow-up and genotyping revealed HPV52 in 2 of four women tested suggesting that HPV52 may be the predominant genotype in Bario. Conclusions The careHPV system is portable and can be used in low resource settings as long as reliable electricity is available. We found that careHPV DNA test is more objective compared to VIA and can be used to screen women outside the recommended eligible age. This is especially true for older women in the rural who have never had any form of cervical cancer screening before.

\section{Introduction}

Cervical cancer is the second most common killer in Malaysian women after breast cancer. Around 1682 women are diagnosed with the disease annually and 944 die from the disease (1). The Ministry of Health, Malaysia has introduced free Pap smear as the primary cervical cancer screening test since the 1960 s, but the national coverage hovers around $22 \%$ (2) and consistently failed to screen the targeted $40 \%$ of the eligible women between age 20-65 (3). The coverage of Pap smear would be significantly lower in rural communities such as in the interior of Sarawak. Coincidentally, Sarawak has the highest cervical cancer incidence rate in Malaysia (4). Although there is an absence of statistical data on the number of new cervical cancer cases reported from the women living in the rural Sarawak compared to women who lived in the urban, it is generally known that the majority of all cervical cancer mortality worldwide are from the communities living in the low resource settings. These patients are usually presented to the healthcare facility with stage II cervical cancer after symptomatic presentation.

Sarawak is located in East Malaysia on the island of Borneo, bordering Kalimantan, Indonesia; the Sultanate of Brunei and Sabah. Is has a population of around 2.4 million, consisting of the indigenous people of Sarawak [Iban (28.9\%); Bidayuh (8.0\%); other Sarawak natives (6.3\%); Melanau (5.0\%), and 
others $(0.7 \%)(5)$. The Kelabits are an ethnic minority group (0.4\%) grouped under the 'other Sarawak natives' or Orang Ulu (People of the Interior in the local language) with the estimated population size of 6000 but about only around 1000 of them remains at their original settlement in the highland of Bario. Other ethnic groups have migrated into Bario either through marriage or work.

Bario is located at $3.7350^{\circ} \mathrm{N}, 115.4793^{\circ} \mathrm{E}$ about $180 \mathrm{~km}$ southeast from the city of Miri (Figure 1) and has an altitude $>1 \mathrm{~km}$ above sea level. There is no road access except a 14 hours off-road journey via the logging trail. Alternatively, the town of Bario is connected by rural air service from the city of Miri and Marudi town via 16 seater twin Otter operated by MasWing, a subsidiary of the national carrier, Malaysia Airlines.

The Kelabits in Bario mainly engaged in farming activities cultivating high-quality Bario rice and pineapples, producing iodine-rich salt from saltwater wells and making traditional handicrafts.

Currently, the communities in Bario enjoy basic healthcare via a health clinic since 2018. The laboratory facility is currently absent at the health clinic. More serious health issues and more complex health screening procedures and laboratory testing are referred to Miri General Hospital. Cervical smear can be done in Bario Health Clinic but the fixed-slides are to be sent to the laboratory in Miri for Papanicolaou (Pap) staining and interpretation. The Pap smear results often return between 2 weeks to 3 months with the risk of being lost en route. Recalling the women for their results can also be a challenge. There is no post office in Bario and the nearest post office is in Miri. Nevertheless, Bario can receive posts via Poslaju, the national courier service provider. The health clinic is currently powered by roof-mounted solar panels but insufficiency is supplemented or taken over by a diesel-powered generator. Cellular and 4G mobile coverage is limited to $1 \mathrm{~km}$ radius from the heart of Bario and has only one service provider.

In 2014, the World Health Organization has recommended both HPV vaccination and primary HPV cervical screening with hrHPV testing to prevent and control cervical cancer (6). HPV vaccination was introduced in 2010 has been incorporated into the National Childhood Immunisation program in 2012 targeting $>250000$ school girls aged 13 years annually (3). The non-target groups can obtain their HPV vaccination privately but both the cost and availability remain a challenge for those living in the low resource settings. It is still too early to speculate if HPV vaccination will reduce the cervical cancer incidence rate. Therefore, effective and penetrative screening strategies are still required to reach the unreachable.

Visual inspection using Acetic acid (VIA) is recommended over Pap smear in the low-resource settings due to its higher sensitivity than cytology, more cost-effective and provides immediate results allowing VIA positive women to be counselled and referred for treatment during the screening itself (7). Nevertheless, VIA has a lower sensitivity compared to HPV DNA detection. With most HPV DNA tests being laboratory-based, collected cervical specimens would have to be sent to the laboratory to be tested and this method may not provide much faster results in the rural. To date, careHPV HPV Test System, a simplified version of the larger Digene HC2 HPV Test System developed by Qiagen is the only portable equipment made to be used in the low resource setting. 
In this study, we evaluate the feasibility of the portable careHPV test system (Qiagen) in the low resource setting in the highland of Bario.

\section{Materials And Methods}

\section{Medical Ethics Approval}

The study was approved by Universiti Malaysia Sarawak Medical Ethics Committee UNIMAS/NC-21. 02/03-02 Jld. 3 (17).

\section{Study design}

This is a cross-sectional study. The medical team consisting of gynecologists, general practitioners, nurses, a virologist, and volunteers travelled to Bario from the city of Kuching via the city of Miri for the 2 days cervical cancer screening program from $21^{\text {st }}-22^{\text {nd }}$ April 2019.

\section{The careHPV system}

The 3-piece careHPV system and a careHPV kit (Qiagen) was procured via Qiagen's preferred distributor in Sarawak.

The careHPV system (Qiagen) together with the packaging weighs around $30 \mathrm{~kg}$ and was brought to Bario by flight as checked-in baggage [Figure 2A]. A total of 90 careBrush and 90 careMedium, Repeater E3 (Eppendorf), 10-200ml pipet (Eppendorf), Combitips (Eppendorf), a box of 200ml long-reach tip (MBP), 6 plate seals (Qiagen), test tube rack (Nalgene), paper towel, a double socket Automatic Voltage Regulator (AVR), a 3-point socket adapter, and an autoclave bag were packed separately. The system was set up in one of the consultation rooms at the Bario Health Clinic No. 10 and the reliability of electricity supplied by the solar panels and diesel generator was evaluated [Figure 2B].

\section{Sensitization}

The purpose of the Cervical cancer outreach program was broadcasted via Radio Bario, a community-run broadcasting station that keeps the isolated highland community connected. The news was also conveyed during church services, by word of mouth, through community leaders and the Bario health clinic at least 2 weeks before the outreach. 


\section{Subjects and specimens collection}

All women who have had at least one sexual encounter and presented to the cervical cancer screening program were offered the HPV test. Those ages $<50$-year-old were offered VIA.

Women who wished to know their HPV status and/or have their cervical examination done but do not wish to be part of the study were screened as well but data obtained from them were excluded in this manuscript.

\section{Cervical sample collection, VIA, and HPV DNA test}

Cervical swabs were clinician-collected using the careBrush (Qiagen) according to the manufacturer's instructions and preserved in careMedium (Qiagen) at ambient temperature. The sample tubes were arranged and labelled corresponding to the coordinate of the 96-well plate to facilitate sample loading as well as to reduce pipetting error (Figure $3 \mathrm{~A}$ ). All of the samples were screened for hrHPV according to the manufacturer's instruction by a certified operator (Figure 3B). The careHPV Test detects the presence of 14 high-risk oncogenic HPV types 16, 18, 31, 33, 35, 39, 45, 51, 52, 56, 58, 59, 66, 68 using full genome probes complementary to HPV DNA, specific DNA, specific antibodies, signal amplification and chemiluminescent detection. Positive results were defined as the relative light unit (RLU) equal to or greater than the cut-off value as measured by the luminometer.

Visual inspection via acetic acid (VIA) was performed for all participants aged $<60$ by two certified VIA providers from Universiti Malaysia Sarawak. Participants aged $>60$ years (peri- or post-menopausal) were not offered VIA as their columnar epithelium and transformation zone would have retreated back from the outer cervix into the endocervical canal (8), reducing the sensitivity and specificity of VIA.

\section{Genotyping}

hrHPV positive participants were recruited and a separate cervical swab was obtained using the Rover Cytobrush (BD) and preserved in Surepath Medium (BD) according to the manufacturer's instruction. Two $\mathrm{mL}$ of the cell suspension was pelleted via centrifugation, Surepath medium removed by aspiration and the cell pellet was resuspended in $200 \mathrm{~mL}$ of phosphate-buffered saline pH7.4.

Viral nucleic acid was extracted using High Pure Nucleic Acid Extraction Kit (Roche) and eluted in 50ml of elution buffer supplied in the kit. Internal control was carried out against the human b-globin gene. Nested-PCR was carried out by using the MY09/MY11 as the outer primers and GP5+/GP6+ as the inner primers (9). Sanger sequencing service was outsourced to Apical Biotechnology (Kuala Lumpur, Malaysia) and the sequences obtained were compared with the sequences in the Genbank for HPV genotype identification. 


\section{Results}

Electricity supply from the diesel-powered generator was more reliable compared to the solar panels. A generator is limited to the supply of diesel but solar energy depends on the uncontrolled changes in the weather. Diesel-powered electricity supply regulated by an automated voltage regulator is more practical and reliable in the low resource settings.

A total of 89 patients were screened and 75 patients have provided informed consent. Patients' age was 27-83 years old (median age 55 years). 20.2\% (18/89) of the samples in careMedium were found to be visually turbid suggesting thrush and the application of these samples seem to coat the microwell producing smearing of the magnetic beads (Figure 4). The significance of this phenomenon is unknown but no other careHPV operators have reported such a phenomenon. Nevertheless, the product information sheet indicated that Candida spp., one of the causative agents for thrush will not interfere with the result.

We detected hrHPV infection in 6 women $(n=6 / 75 ; 8.00 \%)$. Of the 6 women, 5 are Kelabits $(n=5 / 64$; $7.81 \%)$ and one Indonesian $(n=1 / 4 ; 25.00 \%)$. No hrHPV was detected in the Lun Bawang $(n=0 / 1 ; 0.00 \%)$, Penan $(n=0 / 4 ; 0.00 \%)$, and Iban ( $n=0 / 2 ; 0.00 \%)$ (Table 1$)$.

It is worthy to note that both age group of $61-70$ and 71-80 years old have the highest prevalence rate of $2.6 \%$ respectively while age groups $41-50$ and $51-60$ each recorded the same prevalence rate of $1.3 \%$. Although most publications would have suggested the high prevalence among the 20-29 years old but our representative sample size for the younger age group is low $(n=2)$ in-line with the mass migration of the younger generations to larger cities for education and job opportunities.

The six women positive for hrHPV were recalled for follow-up. Only four of them responded to the call for follow-up and have consented for additional cervical swabs to be obtained. Of the 4 cervical swabs, all were positive for b-globin gene but only 2 of them were positive for HPV via nested-PCR. Both sequences matched HPV52 in the Genbank.

Table 1 hrHPV and VIA status among women in Bario according to Ethnic group. ( $n=75)$

\begin{tabular}{|l|r|r|r|r|r|r|}
\hline \multirow{2}{*}{ Ethnic Group } & \multicolumn{3}{|c|}{ hrHPV } & \multicolumn{3}{|c|}{ VIA } \\
\cline { 2 - 7 } & Negative & Positive & Total & Negative & Positive & Total \\
\hline Kelabit & 51 & 5 & 56 & 19 & 5 & 24 \\
\hline Indonesian & 2 & 1 & 3 & 2 & 1 & 3 \\
\hline Penan & 2 & 0 & 2 & 2 & 1 & 3 \\
\hline Lun Bawang & 1 & 0 & 1 & 1 & 0 & 1 \\
\hline Iban & 2 & 0 & 2 & 2 & 0 & 2 \\
\hline Total & 69 & 6 & 75 & 26 & 7 & 33 \\
\hline
\end{tabular}

Table 2 hrHPV and VIA status among the women in Bario according to Age group $(n=75)$. 


\begin{tabular}{|c|c|c|c|c|c|c|}
\hline \multirow{2}{*}{ Age Group (Years) } & \multicolumn{3}{|c|}{ hrHPV } & \multicolumn{3}{c|}{ VIA } \\
\cline { 2 - 7 } & Negative & Positive & Total & Negative & Positive & Total \\
\hline $21-30$ & 3 & 0 & 3 & 3 & 0 & 3 \\
\hline $31-40$ & 10 & 0 & 10 & 9 & 1 & 10 \\
\hline $41-50$ & 17 & 1 & 18 & 12 & 6 & 18 \\
\hline $51-60$ & 17 & 1 & 18 & 2 & 0 & 2 \\
\hline $61-70$ & 8 & 2 & 10 & - & - & - \\
\hline $71-80$ & 10 & 2 & 12 & - & - & - \\
\hline $81-90$ & 4 & 0 & 4 & - & - & - \\
\hline TOTAL & 69 & 6 & 75 & 26 & 7 & 33 \\
\hline
\end{tabular}

\section{Discussion}

The packaging and transportation of the careHPV system was straightforward leaving up to its claim to be portable. However, the system which was introduced since 2013 can be further improved to reduce the weight and profile to facilitate transportation to the geographically challenging locations. The installation of the 3-piece careHPV system was simple and took less than 20 min including unpacking. However, reliable electricity remains a challenge as the blank trial run was failed due to insufficient power supplied by the solar panels. Although the power from the generator kicked in minutes later, the careHPV system by default restarted itself rather than to continue from where it had stopped. This experience was reported by Trope et al. , 2013 which has caused the loss of a batch test in Roi-et, Thailand (10). It is interesting to note that some authors have reported that the careHPV system can be operated without an external power source (11-13) as the careHPV system does not come with built-in batteries.

Nevertheless, the authors did not elaborate further on how they have operated their careHPV system without electricity.

The careHPV DNA test has been prequalified by WHO since 2018 and boasted for the affordable cost per test of USD\$5 in China and Thailand $(11,14-16)$. The actual cost per test as per our experience in Sarawak, Malaysia is a few times more than USD $\$ 5$ but slightly lower compared to the cost reported by the researchers in Myanmar (17). The careHPV cost per test is therefore not USD\$5 across the globe and should not be regarded as universal. For instance, Campos and colleagues have used this 'universal' cost per test to calculate the cost-effectiveness of the point-of-care HPV screening in India, Nicaragua, and Uganda (18) and have been cited elsewhere giving the incorrect perception that the cost per test for careHPV is fixed at USD $\$ 5$ everywhere in the world. In actual fact, the cost per test for careHPV is largely unregulated and the price for careHPV varies from country to country (19).

The careHPV test's turnaround time was slightly more than 3 hours, 30 min longer than the ideal 2.5 hours as claimed by the manufacturer but 3 to $3.5 \mathrm{~h}$ turnaround time is more realistic (19). Some discrepancies were also noted within the manufacturer's claims. For instance, Qiagen's careHPV product profile states 'convenient test results in 2.5 hours for possible same-day follow-up'(20) but its WHO Prequalification of In Vitro Diagnostics Public Report states 'an approximate time to result of 3.5 hours' (21). However, a shorter turnaround time of $2 \mathrm{~h}$, which is 30 mins faster than the instrument time has also 
been reported (15) but we find that it is impossible even to achieve a turnaround time of 2.5 hours. The real-life turnaround time is crucial if same-day result is desired. Assuming that we are following the Malaysian government's official working hour, all cervical-sample collection activity must end before the lunch break at $1 \mathrm{pm}$ and the test must commence immediately in order for the results to be released around $4 \mathrm{pm}$. This is to allow time for the clinician to see the hrHPV positive patients for a referral or to obtain the second swab for HPV genotyping. In an ideal situation, participants may submit their selfcollected cervicovaginal swabs to the test center early in the morning (or a day before) and the batch test can start around 9 in the morning and the participants may come and collect their results after lunch break, and having ample of time for consultation, treatment or referral.

The hrHPV prevalence rate in the population in Bario is $8.00 \%$ which matches the estimated hrHPV prevalence rate in Asia (22). However, the prevalence rate is significantly lower than the prevalence rate in large cities around this region such as Johore and Singapore $(n=2364$; prevalence rate $=25.6 \%)(23)$ and Southern Selangor $(n=1153$; prevalence rate $=46.7 \%)(9)$. Nevertheless, Bario represents a remote town that lacks accessibility with a relatively closed community and the best hrHPV prevalence rate comparison would be with the remote districts in Nepal which have reported the prevalence rates between 8.6-14.4\% (24). It is noteworthy that our experimental design was based on opportunistic sampling without any age limit. This has contributed to the detection of $4 \mathrm{hrHPV}$ cases in the 70-79 year group. This group of women would have otherwise be excluded from the national cervical cancer screening program's upper age limit of 65-year. If we would have specified the age range of the participants according to the national guideline, the hrHPV prevalence would be much lower at $2.67 \%(2 / 75)$ from the age range between 40-59. To date, the lowest published hrHPV prevalence rate is among the Turkish women $(2.79 \% ; n=11624)(25)$. The very low hrHPV prevalence may be contributed by the socio-cultural characteristics and widespread monogamy in Bario's population.

The prevalence rate of hrHPV among the Kelabit women in Bario is $7.94 \%$ and this is the first hrHPV prevalence data obtained for the geographically-isolated ethnic group in Sarawak, Malaysia. Two Kelabit women were positive for HPV52. Although the sample is small, this may suggest that HPV52 is the predominant hrHPV in the community Bario (at least 33.3\%). No other HPV genogroup was detected in this study nor can we ruled out the possibility of co-infection by more than one HPV genotype, which is the known limitation of the genotyping method used in this study. Researchers in China have also reported that HPV52 is the predominant endemic HPV genotype $(26,27)$ and the prevalence of endemic HPV genotypes in other parts of Sarawak needs to be elucidated. The baseline data for the prevalence of endemic HPV genotypes are important to influence the adoption or development of relevant HPV vaccine.

The requirement for additional swabs to be obtained is due to the incompatibility of careMedium with genomic DNA extraction and PCR. Efforts were made to consult the manufacturer but they offered no explanation. Improving the careMedium formulae to make it compatible with common DNA extraction methods and PCR would eliminate the need to obtain a separate swab for HPV genotyping. After all, the careHPV test only uses $50 \mathrm{~mL}$ of the suspended exfoliated cervical cells in careMedium and the remaining $950 \mathrm{~mL}$ which still holds precious biological information would literally go to waste. 
Of the six hrHPV positive women, 4 returned to the clinic for subsequent follow-up while the remaining two have returned to their respective villages and defaulted on their appointments. As with the limitation of telecommunication in Bario, the two defaulters cannot be contacted. Those hrHPV positive women would eventually need to undergo colposcopy at the Miri General Hospital and this would post both logistic and financial constraint to the women; bearing in mind that Miri and Bario are $180 \mathrm{~km}$ apart and the cost of living in Miri bordering the oil-rich sultanate of Brunei is not helping either. We foresee that all of these hrHPV women would eventually default on their follow-ups unless external assistance is available. All these can be prevented by employing the screen-and-threat programs (28) as multiple visits for diagnosis and treatment have been consistently shown to be counter-effective (29). Since we already have a robust careHPV system, the acquisition of a portable colposcope and treatment device $(30,31)$ would allow us to carry out the screen-and treat program.

$\mathrm{N}=5 / 6$ of the women tested positive for hrHPV was VIA -ve. These may indicate the clinical sensitivity and specificity of careHPV test are relatively high and low respectively. $\mathrm{N}=6$ women were negative for hrHPV but were VIA positive. This phenomenon may indicate that the cervical intraepithelial neoplasia (CIN) graded via VIA may not be indicative of true pre-cancerous lesion.

\section{Conclusion}

The careHPV system is robust and can be used in the resource-limited settings in the interior of Sarawak as long as a reliable external power source is available. The prevalence rate of hrHPV among the women screened in Bario is $8 \%$ and $7.81 \%$ among the Kelabit women which is similar to the estimated prevalence rate in Asia. HPV52 was detected in two Kelabit women, an HPV genotype that is absent in the current bivalent and quadrivalent HPV vaccines.

\section{Declarations}

\section{Ethical Approval and Consent to participate}

The study was approved by Universiti Malaysia Sarawak Medical Ethics Committee UNIMAS/NC-21. 02/03-02 Jld. 3 (17). Standard procedures were followed to obtain informed consent from each participant before undergoing the procedures.

\section{Consent for publication}

The results obtained from those who have provided their consent for publication were included in this manuscript. Participants who wished to undergo cervical cancer screening but refused to consent for publication were provided with the same medical care but the results obtained from them were excluded from this manuscript.

\section{Availability of supporting data}


The data generated during this study are not publicly available, but a transcribed file is available from the corresponding author Cheng-Siang Tan (cstan@unimas.my) on reasonable request.

\section{Competing interests}

The authors declared that they have no competing interests.

\section{Funding}

Sarawak State Legislative Assembly of Sarawak (Dewan Undangan Negeri Sarawak) N78 Mulu funded the logistics

Pink and Teal Empowher, a NGO raised the fund for the careHPV test via the 'Sigek kitak sigek kamek' (One for you and one for me) campaign organized by Pink and Teal Empowher. In this campaign, for every HPV test purchased, one test will be donated to the in the rural.

The Ministry of Education Malaysia, Special Grant Scheme (F05/SpGS/1564/2017) funded the other HPV genotyping and other consumables.

\section{Authors' contributions.}

ARJ and MK obtained the informed consent, performed the cervical examination, obtained the cervical swabs, and critically reviewed and provided clinical input to the manuscript.

CST performed the HPV DNA test using the careHPV system, HPV genotyping, and wrote the manuscript.

\section{Acknowledgements}

The cervical cancer screening program is the inaugural medical outreach program under the of the Sarawak $\underline{\text { Urban }}$ And Rural Action for Cervical Cancer Prevention (SUARA) initiative that was initiated by Pink and Teal Empowher (an NGO) in collaboration with Universiti Malaysia Sarawak (UNIMAS).

We want to thank Sarawak Health Department and Health Clinic No.10, Bario, and volunteers who have supported us. This manuscript will not be possible without them.

Special thanks to Dr Nadia Diyana who have follow-up with the four hrHPV positive women, obtained additional informed consent for a second swab to be obtained for HPV genotyping.

\section{Authors' information}

Dr Cheng Siang Tan

Centre for Tropical and Emerging Diseases, Faculty of Medicine and Health Sciences, Universiti Malaysia Sarawak, 94300 Kota Samarahan, Sarawak, Malaysia 
Department of Obstetrics and Gynaecology, Faculty of Medicine and Health Sciences, Universiti Malaysia Sarawak, 94300 Kota Samarahan, Sarawak, Malaysia

Dr Mardiana Kipli

Department of Obstetrics and Gynaecology, Faculty of Medicine and Health Sciences, Universiti Malaysia Sarawak, 94300 Kota Samarahan, Sarawak, Malaysia

Corresponding author: Cheng Siang Tan

cstan@unimas.my

\section{References}

1. Human Papillomavirus and Related Diseases Report ASIA [Internet]. ICO/IARC Information Centre on HPV and Cancer (HPV Information Centre). 2018 [cited 2018 May 16]. Available from: http://www.hpvcentre.net/statistics/reports/XSX.pdf

2. Yunus NA, Mohamed Yusoff H, Draman N. Non-Adherence to recommended Pap smear screening guidelines and its associated factors among women attending health clinic in Malaysia. Malaysian Fam physician Off J Acad Fam Physicians Malaysia [Internet]. 2018 [cited 2019 Jun 14];13(1):10-7. Available from: http://www.ncbi.nlm.nih.gov/pubmed/29796205

3. Buang SN, Ja'afar S, Pathmanathan I, Saint V. Human papillomavirus immunisation of adolescent girls: improving coverage through multisectoral collaboration in Malaysia. BMJ [Internet]. 2018 Dec 7 [cited 2019 Jul 18];363:k4602. Available from: http://www.ncbi.nlm.nih.gov/pubmed/30530626

4. Manan A, Nor Saleha IT, Abdullah NH, Asmah ZA, Wahab M. Malaysian National Cancer Registry Report 2007-2011 [Internet]. National Cancer Institute, Ministry of Health Malaysia. 2015 [cited 2019 Jun 24]. Available from:

https://drive.google.com/file/d/1pCsJlw0ysFccCMFA0XqzfbYu1OFDRbO_/view

5. Utap MS, Kiyu A. Active case detection of leprosy among indigenous people in Sarawak, East Malaysia [Internet]. [cited 2019 Jun 14]. Available from: https://www.lepra.org.uk/platforms/lepra/files/lr/Dec17/Lep563-567.pdf

6. World Health Organisation. WHO | Guidelines for the prevention and control of cervical cancer [Internet]. WHO. World Health Organization; 2016 [cited 2019 Jun 14]. Available from: https://www.who.int/reproductivehealth/topics/cancers/hpv-vaccination/en/

7. Path. Cervical cancer screening and treatment in low-resource settings practical experience from path | 2013 Cervical Cancer Prevention: Practical Experience Series acknowledgments [Internet]. 2013 [cited 2019 Jul 18]. Available from: https://path.azureedge.net/media/documents/RH_ccp_screening_treatment.pdf

8. WHO. Comprehensive Cervical Cancer Control [Internet]. WHO; 2006 [cited 2019 Jul 18]. Available from: www.rho.org/HPV-screening-treatment.htm 
9. Chong PP, Hassan F, Latiff LA. High Prevalence of Human Papillomavirus DNA Detected in Cervical Swabs from Women in Southern Selangor, Malaysia. Asian Pacific J Cancer Prev [Internet]. 2010 [cited 2019 Jun 14];11:1645-51. Available from:

https://www.researchgate.net/publication/49852326

10. Trope LA, Chumworathayi B, Blumenthal PD. Feasibility of Community-Based careHPV for Cervical Cancer Prevention in Rural Thailand. J Low Genit Tract Dis [Internet]. 2013 Jul [cited 2019 May 1];17(3):315-9. Available from: http://content.wkhealth.com/linkback/openurl? sid=WKPTLP:landingpage\&an=00128360-201307000-00013

11. Tambouret R. Screening for Cervical Cancer in Low-Resource Settings in 2011. Arch Pathol Lab Med [Internet]. 2013 [cited 2019 Jun 22];137:782-90. Available from: www.cervicalcanceraction.org;

12. Ying H, Jing F, Fanghui Z, Youlin Q, Yali H. High-risk HPV nucleic acid detection kit-the careHPV test -a new detection method for screening. Sci Rep [Internet]. 2015 May 16 [cited 2019 Jun 23];4(1):4704. Available from: http://www.nature.com/articles/srep04704

13. Lorenzi AT, Fregnani JHTG, Possati-Resende JC, Neto CS, Villa LL, Longatto-Filho A. Self-collection for high-risk HPV detection in Brazilian women using the careHPV ${ }^{\mathrm{TM}}$ test. Gynecol Oncol [Internet]. 2013 Oct 1 [cited 2018 Aug 23];131(1):131-4. Available from:

https://www.sciencedirect.com/science/article/pii/S0090825813010123

14. Kang L-N, Jeronimo J, Qiao Y-L, Zhao F-H, Chen W, Valdez M, et al. Optimal positive cutoff points for careHPV testing of clinician- and self-collected specimens in primary cervical cancer screening: an analysis from rural China. J Clin Microbiol [Internet]. 2014 Jun 1 [cited 2019 Jun 22];52(6):1954-61. Available from: http://www.ncbi.nlm.nih.gov/pubmed/24671789

15. Levin CE, Sellors J, Shi J-F, Ma L, Qiao Y, Ortendahl J, et al. Cost-effectiveness analysis of cervical cancer prevention based on a rapid human papillomavirus screening test in a high-risk region of China. Int J Cancer [Internet]. 2010 Jan 4 [cited 2019 Jun 22];127(6):1404-11. Available from: http://doi.wiley.com/10.1002/ijc.25150

16. Kang L-N, Castle PE, Zhao F-H, Jeronimo J, Chen F, Bansil P, et al. A prospective study of age trends of high-risk human papillomavirus infection in rural China. BMC Infect Dis [Internet]. 2014 Dec 21 [cited 2019 Jul 19];14(1):96. Available from: http://bmcinfectdis.biomedcentral.com/articles/10.1186/1471-2334-14-96

17. Tin-Oo C, Hlaing HNT, Nandar CS, Aung T, Fishbein D. Why the Cost of Purchasing the careHPV Test in Myanmar Was Many Times Greater Than That Reported in the International Literature. J Glob Oncol [Internet]. 2018 Oct 28 [cited 2019 Jun 22];(4_suppl_2):49s-49s. Available from: http://ascopubs.org/doi/10.1200/jgo.18.27900

18. Campos NG, Tsu V, Jeronimo J, Mvundura M, Kim JJ. Estimating the value of point-of-care HPV testing in three low- and middle-income countries: a modeling study. BMC Cancer [Internet]. 2017 Dec 25 [cited 2019 Jun 22];17(1):791. Available from: http://www.ncbi.nlm.nih.gov/pubmed/29178896

19. Qiao Y lin, Sellors JW, Eder PS, Bao Y ping, Lim JM, Zhao F hui, et al. A new HPV-DNA test for cervical-cancer screening in developing regions: a cross-sectional study of clinical accuracy in rural 
China. Lancet Oncol. 2008;9(10):929-36.

20. careHPV Test Kit - QIAGEN Online Shop [Internet]. [cited 2019 Jul 16]. Available from: https://www.qiagen.com/ie/products/diagnostics-and-clinical-research/sexual-reproductivehealth/cervical-cancer-screening/carehpv-test-kit/\#orderinginformation

21. WHO Prequalification of In Vitro Diagnostics [Internet]. 2018 [cited 2019 Jul 16]. Available from: https://www.who.int/diagnostics_laboratory/evaluations/pq-

list/180713_pqpr_pqdx_0085_028_00_carehpv_with_labelling.pdf?ua=1

22. de Sanjosé S, Diaz M, Castellsagué X, Clifford G, Bruni L, Muñoz N, et al. Worldwide prevalence and genotype distribution of cervical human papillomavirus DNA in women with normal cytology: a meta-analysis. Lancet Infect Dis [Internet]. 2007 Jul 1 [cited 2019 Jul 15];7(7):453-9. Available from: http://www.ncbi.nlm.nih.gov/pubmed/17597569

23. TAY S-K, TAY Y-K. The prevalence and significance of high-risk human papillomavirus DNA test in southern Malaysia and Singapore. Aust New Zeal J Obstet Gynaecol [Internet]. 2009 Jun 1 [cited 2019 Jun 14];49(3):323-7. Available from: http://doi.wiley.com/10.1111/j.1479-828X.2009.01000.x

24. Thapa N, Maharjan M, Shrestha G, Maharjan N, Petrini MA, Zuo N, et al. Prevalence and type-specific distribution of human papillomavirus infection among women in mid-western rural, Nepal- $\mathrm{A}$ population-based study. BMC Infect Dis [Internet]. 2018 Dec 20 [cited 2019 Jul 15];18(1):338. Available from: https://bmcinfectdis.biomedcentral.com/articles/10.1186/s12879-018-3175-9

25. Kulhan M, Kulhan NG, Seven Y, Nayki UA, Nayki C, Ata N, et al. Estimation of the prevalence and distribution of HPV genotypes and identification of related risk factors among Turkish women. Contemp Oncol (Poznan, Poland) [Internet]. 2017 [cited 2019 Jul 15];21(3):218-23. Available from: http://www.ncbi.nlm.nih.gov/pubmed/29180929

26. Xu HH, Lin A, Chen YH, Dong SS, Shi WW, Yu JZ, et al. Prevalence characteristics of cervical human papillomavirus (HPV) genotypes in the Taizhou area, China: a cross-sectional study of 37967 women from the general population. BMJ Open [Internet]. 2017 [cited 2019 Jul 15];7(6):e014135. Available from: http://www.ncbi.nlm.nih.gov/pubmed/28601819

27. Wang X, Zeng Y, Huang X, Zhang Y. Prevalence and Genotype Distribution of Human Papillomavirus in Invasive Cervical Cancer, Cervical Intraepithelial Neoplasia, and Asymptomatic Women in Southeast China. Biomed Res Int [Internet]. 2018 Oct 8 [cited 2019 Sep 11];2018:1-10. Available from: https://www.hindawi.com/journals/bmri/2018/2897937/

28. Klein G, Pack A, Reuter G. Antibiotic resistance patterns of enterococci and occurrence of vancomycin-resistant enterococci in raw minced beef and pork in Germany. Appl Environ Microbiol [Internet]. 1998 May [cited 2017 Aug 12];64(5):1825-30. Available from: http://www.ncbi.nlm.nih.gov/pubmed/9572958

29. Sankaranarayanan R, Esmy PO, Rajkumar R, Muwonge R, Swaminathan R, Shanthakumari S, et al. Effect of visual screening on cervical cancer incidence and mortality in Tamil Nadu, India: a clusterrandomised trial. Lancet [Internet]. 2007 Aug 4 [cited 2019 Jul 18];370(9585):398-406. Available from: https://www.sciencedirect.com/science/article/pii/S0140673607611957 
30. Castle PE, Murokora D, Perez C, Alvarez M, Quek SC, Campbell C. Treatment of cervical intraepithelial lesions. Int J Gynecol Obstet [Internet]. 2017 Jul [cited 2019 Jul 18];138:20-5. Available from: http://doi.wiley.com/10.1002/ijgo.12191

31. Path. Treatment Technologies for precancerous cervical lesions Treatment technologies for precancerous cervical lesions in low-resource settings: review and evaluation [Internet]. PATH; 2013 [cited 2019 Jul 18]. Available from: http://www.rho.org/files/rb5/Treatment_technologies_ precancerous_cx_lesions_PATH_2013

\section{Figures}

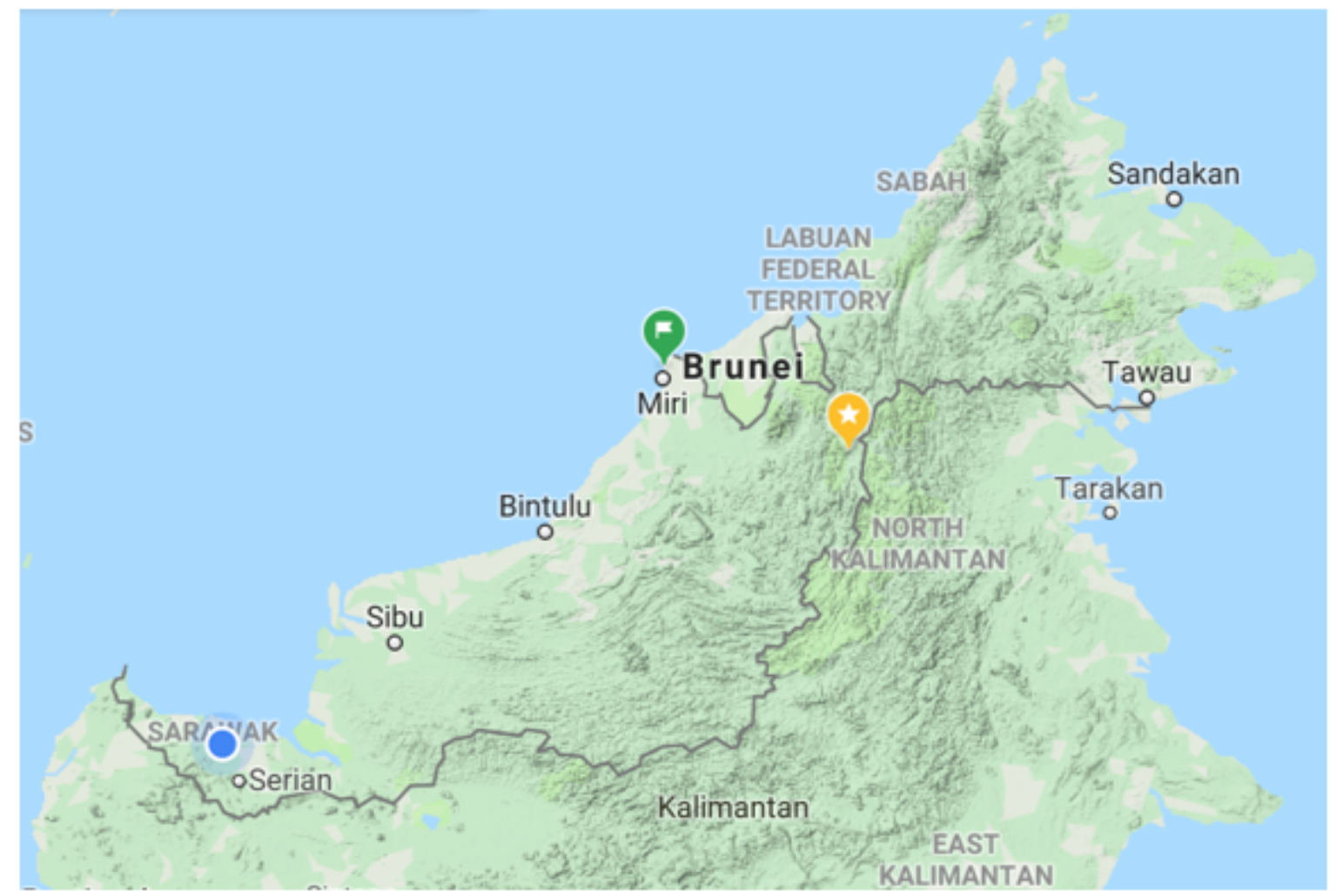

\section{Figure 1}

Borneo Island showing East Malaysia (Sarawak and Sabah), The Sultanate of Brunei and partial Kalimantan, Indonesia. Universiti Malaysia Sarawak is located in Kota Samarahan (blue dot), City of Miri (green flag) and The highland of Bario (Yellow star) [Image from Google Map]. 
A

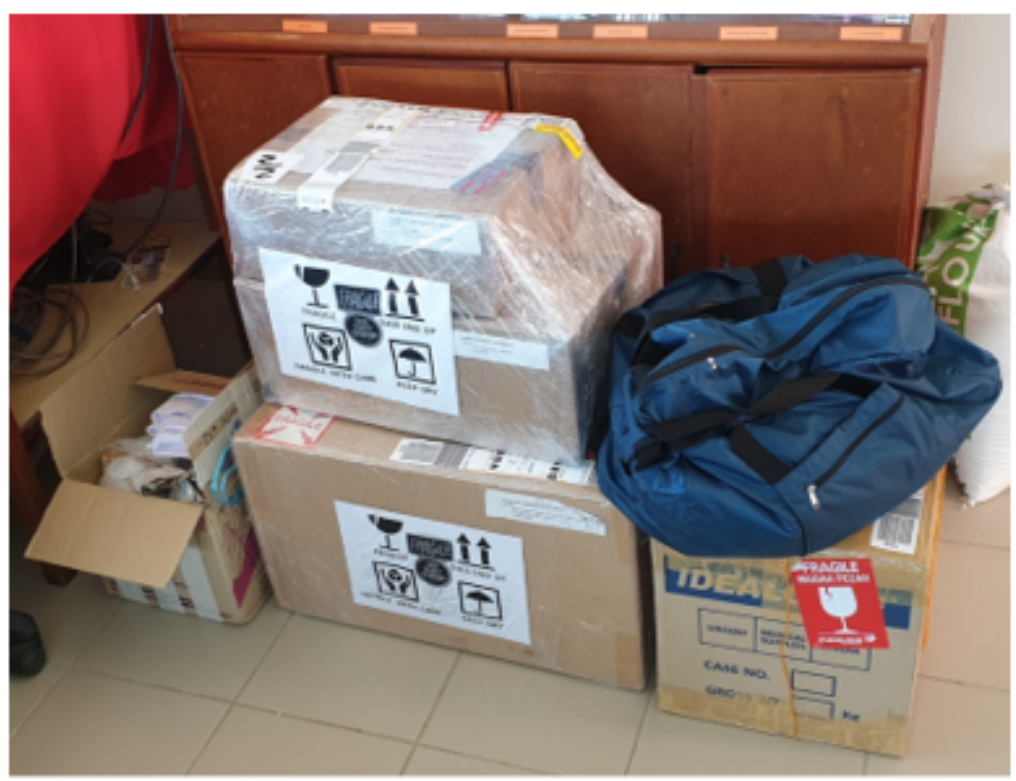

B

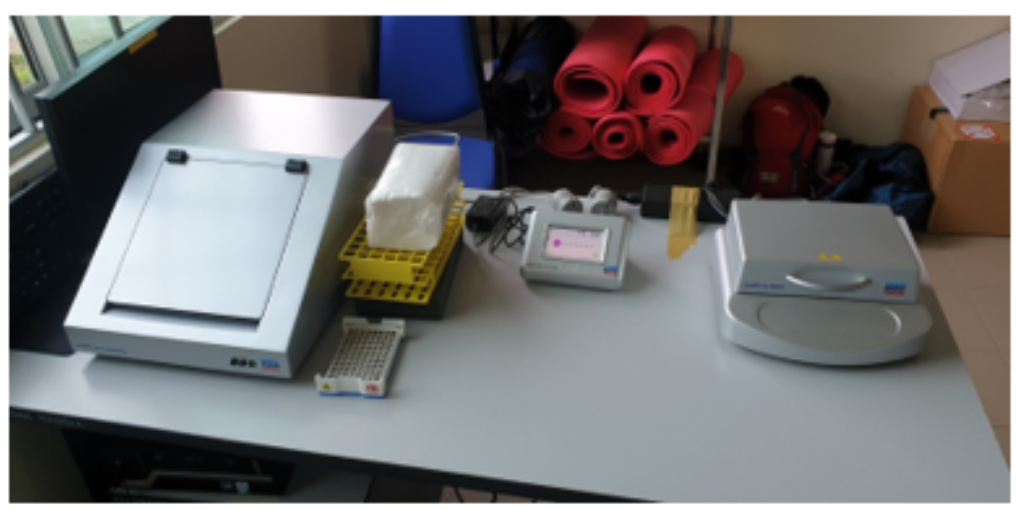

\section{Figure 2}

The careHPV system. (A) The 3-piece careHPV system, reagents, and consumables were packed and transported as check-in baggage. (B) the set-up of the careHPV system at the health clinic. 
A

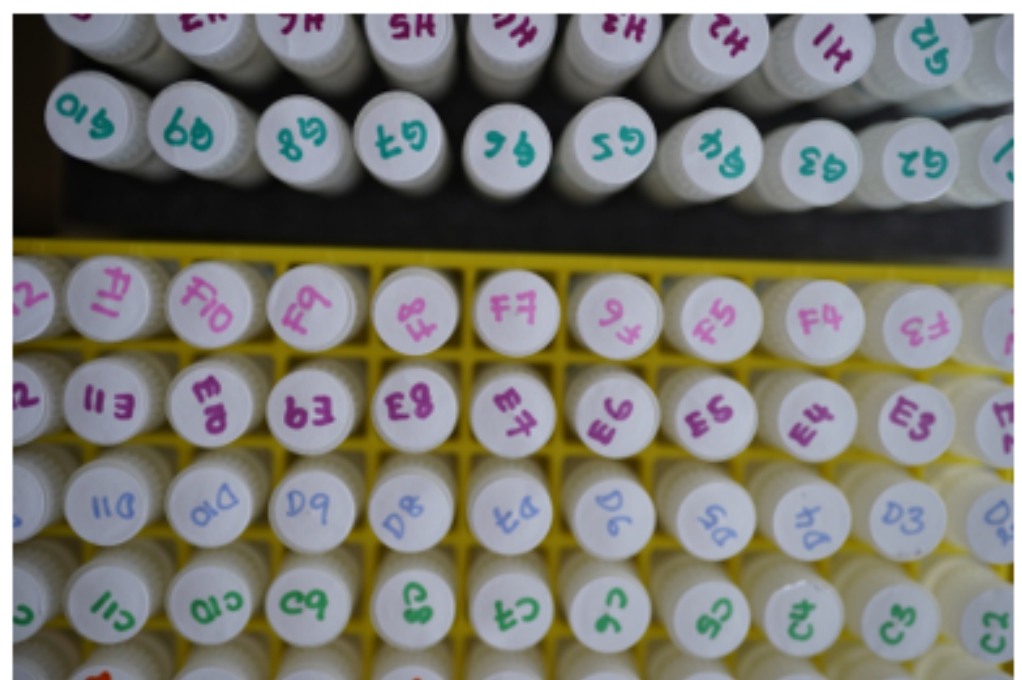

B

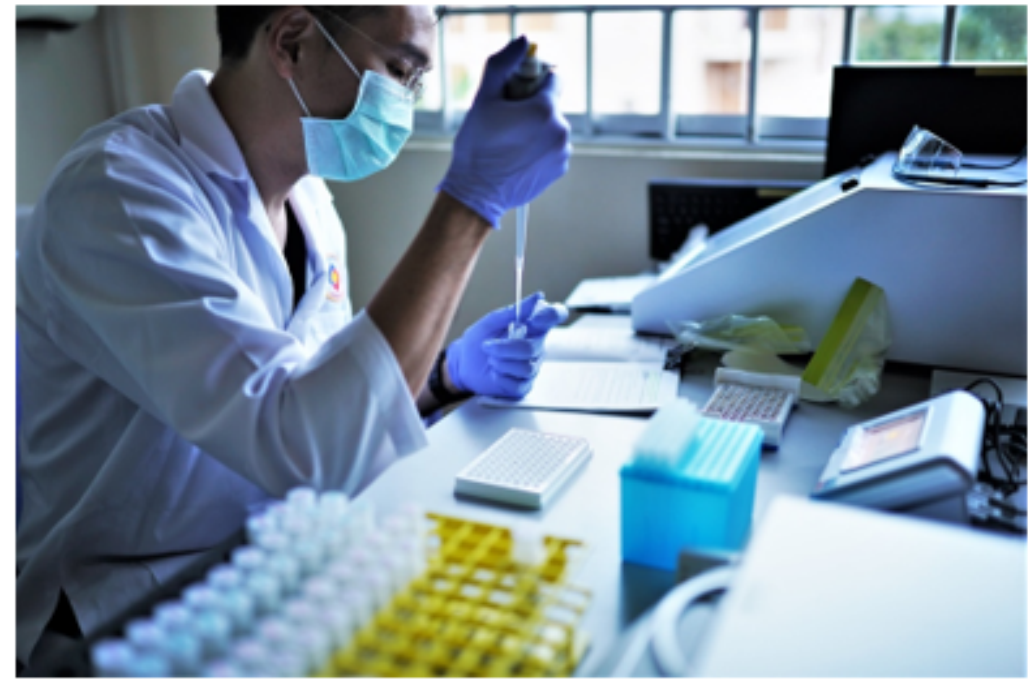

\section{Figure 3}

The careHPV DNA Test. A. Labelling the specimens according to the coordinate of the 96-well plate to facilitate dispensing and reduce pipetting error, B. the certified operator performing the careHPV DNA test.

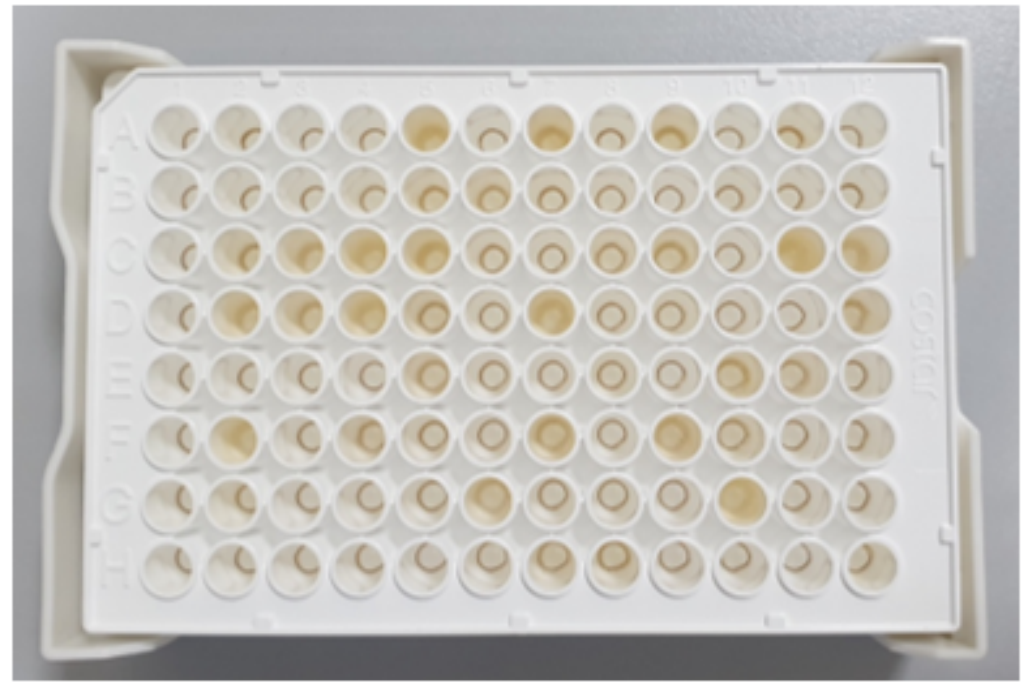

Figure 4 
Smearing effect by turbid samples. Image was taken at the beginning of Protocol 4 of the careHPV DNA test after the magnetic adsorption of the beads and decanting of the supernatant. There is a total of 7 steps in the careHPV DNA Test. 